\title{
Cx32 reverses epithelial-mesenchymal transition in doxorubicin-resistant hepatocellular carcinoma
}

\author{
MEILING YU ${ }^{1}$, GUANGSHU HAN ${ }^{3}$, BENQUAN QI ${ }^{4}$ and XIAOXIANG WU ${ }^{2}$ \\ ${ }^{1}$ Department of Pharmacy, The First Affiliated Hospital of Bengbu Medical College, Anhui, Bengbu 233004; \\ ${ }^{2}$ Department of Pharmacy, The Second Affiliated Hospital of Bengbu Medical College, Anhui, Bengbu 233004; \\ ${ }^{3}$ Faculty of Pharmacy, Bengbu Medical College, Anhui, Bengbu 233030; ${ }^{4}$ Department of Emergency Internal Medicine, \\ The First Affiliated Hospital of Bengbu Medical College, Anhui, Bengbu 233004, P.R. China
}

Received November 2, 2016; Accepted November 30, 2016

DOI: $10.3892 /$ or.2017.5462

\begin{abstract}
Recently, epithelial-mesenchymal transition (EMT) has been reported to be an important mechanism of drug resistance in numerous types of cancer cells, including hepatocellular carcinoma (HCC). However, the underlying mechanisms remain to be fully elucidated. Connexin (Cx)32 plays a crucial role in hepatocarcinogenesis. The present study investigated the role of Cx32 in the regulation of chemotherapyinduced EMT in HCC. We found that the expression levels of Cx32 and E-cadherin were clearly decreased in HCC tissues compared with the corresponding paracancerous tissues, while the expression level of vimentin was significantly enhanced in HCC tissues. The expression of Cx32 had a strong correlation with the expression of E-cadherin and vimentin. In an in vitro study, a doxorubicin (DOX)-resistant liver cell line HepG2/DOX was established from parental HepG2 cells. The results showed that HepG2/DOX cells acquired EMT characteristics, with a decreased expression level of E-cadherin and an enhanced expression level of vimentin, and possessed high migratory abilities and invasiveness. Meanwhile, Cx32 was significantly decreased in the HepG2/DOX cells. Knockdown of Cx32 by shRNA in HepG2 cells induced EMT, while overexpression of Cx32 converted EMT to mesenchymal-epithelial transition (MET) in the HepG2/DOX cells. These results suggest that $\mathrm{Cx} 32$ is an important regulator of DOX-induced EMT in HCC. Cx32 could be considered as a novel target to reverse DOX resistance in $\mathrm{HCC}$.
\end{abstract}

\section{Introduction}

Hepatocellular carcinoma (HCC) is the one of the most common human types of cancer with a high mortality rate

Correspondence to: Dr Xiaoxiang Wu, Department of Pharmacy, The Second Affiliated Hospital of Bengbu Medical College, Anhui, Bengbu 233004, P.R. China

E-mail: 345056979@qq.com

Key words: Cx32, doxorubicin, epithelial-mesenchymal transition, hepatocellular carcinoma cells worldwide (1). To date, surgical resection is still the best option for early-stage patients. However, most patients at the time of diagnosis are in the late stages. Thus, chemotherapy and radiation therapy are the main therapies for patients who are not suitable candidates for surgery (2). Doxorubicin (DOX) is a widely used chemotherapy agent for the clinical treatment of liver cancer. However, during clinical treatment, the development of drug resistance limits the application of $\operatorname{DOX}(3,4)$. Thus, it is necessary to investigate the underlying mechanism of drug resistance of HCC to DOX.

Studies suggest that the occurrence of epithelial-mesenchymal transition (EMT) has an intimate correlation with the process of tumor resistance (5-8). EMT is a special physiological process whereby epithelial cells transform into motile mesenchymal cells, with the downregulation of epithelial characteristics (E-cadherin), the enhancement of mesenchymal characteristics (vimentin, N-cadherin, Snail and Slug) and increased abilities of invasion and migration.

Connexins (Cxs) have been revealed to be proteins which form gap junction channels and mediate the communication between cells (9-11). The intercellular communication is necessary for managing various pathological processes of the human body, such as cell proliferation, differentiation, tissue homeostasis and wound healing (12). Cxs decrease significantly during the process of carcinogenesis and when cancer cells acquire drug resistance. Studies have indicated that $\mathrm{Cx}$ genes are tumor-suppressor genes (13-15) and that recovering or increasing $\mathrm{Cx}$ expression is a new strategy that may be used for cancer therapy.

In the present study, we found that the DOX-resistant human liver cancer HepG2 cell line (HepG2/DOX) exhibited EMT characteristics and that the expression level of Cx32 was gradually decreased after HepG2 cells became resistant to DOX. We also demonstrated that $\mathrm{Cx} 32$ regulated EMT in HepG2 and HepG2/DOX cells. By detecting the expression levels of $\mathrm{Cx} 32$, E-cadherin and vimentin in HCC and the corresponding paracancerous tissues, the results revealed that the expression levels of $\mathrm{Cx} 32$ and E-cadherin were clearly decreased in HCC tissues when compared with the levels in the corresponding paracancerous tissues, while the expression of vimentin was significantly enhanced in the HCC tissues. Moreover, the expression of $\mathrm{Cx} 32$ had a strong correlation 
with the expression of E-cadherin and vimentin. These results suggest that $\mathrm{Cx} 32$ is an important regulator of DOX-induced EMT in HCC.

\section{Materials and methods}

Immunohistochemical samples. HCC and paracancerous tissue specimens were collected from $40 \mathrm{HCC}$ patients who underwent surgical resection at the Hepatobiliary Surgical Department of The First Affiliated Hospital of Bengbu Medical College (Bengbu, China) from February 2014 to June 2015. Paracancerous tissue was the non-cancerous liver tissue located $3-5 \mathrm{~cm}$ from the primary tumor. No patients received radiotherapy, chemotherapy, immunotherapy or other related treatment before surgery. For all patients, complete clinical and pathological data were obtained. All specimens were fixed with neutral formalin solution, paraffin-embedded and continuously sectioned to a $5-\mu \mathrm{m}$ thickness. The specimen collection protocol was approved by the Medical Ethics Committee of the Bengbu Medical College.

Immunohistochemistry. Specimens were embedded in $10 \%$ paraffin and cut by a microtome into $5-\mu \mathrm{m}$ sections. All paraffin sections were routinely deparaffinized and rehydrated in xylene, and graded ethanol solutions. Endogenous peroxidase activity was blocked by $0.3 \% \mathrm{H}_{2} \mathrm{O}_{2}$ in methanol for $20 \mathrm{~min}$. Then, the sections were incubated with the primary antibodies [Cx32 (1:100), E-cadherin (1:400) and vimentin (1:100); Cell Signaling Technology, Danvers, MA, USA] at $4^{\circ} \mathrm{C}$ overnight. Subsequently, the sections were exposed to the biotin-labeled secondary antibody for $20 \mathrm{~min}$. Immunostaining was visualized with 3,3'-diaminobenzidine (DAB) and hematoxylin counterstaining. Phosphate-buffered saline (PBS) instead of a primary antibody was used as the negative control. Positive staining was observed and images were captured with an optical microscope. The stained cells were scored as follows: negative (-), stained cells $<5 \%$; positive (+), stained cells $5-10 \%$; positive $(++)$, stained cells $>10-50 \%$; positive $(+++)$, stained cells $>50-75 \%$; positive $(++++)$, stained cells $>75 \%$.

Materials. The HepG2 cell line was obtained from Shanghai Bai Li Biological Technology Co., Ltd. (Shanghai, China). Cell culture reagents were obtained from Invitrogen (Carlsbad, CA, USA). DOX and dimethyl sulfoxide (DMSO) were obtained from Sigma-Aldrich (St. Louis, MO, USA). Antibodies for E-cadherin, vimentin, $\beta$-actin and the secondary antibodies were obtained from Cell Signaling Technology. shRNA-Cx32, pcDNA-Cx32 and Lipofectamine ${ }^{\mathrm{TM}} 2000$ were obtained from Shanghai GenePharma Co., Ltd. (Shanghai, China).

MTT assay. Cell viability was evaluated by an MTT assay as previously described (16). The cell inhibition rate was calculated to determine the standard pharmacological activity at OD490 $\mathrm{nm}$. Briefly, cells were harvested and seeded in 96-well plates. Various concentrations of DOX were added into the wells followed by $24 \mathrm{~h}$ of incubation $\left(\right.$ at $37^{\circ} \mathrm{C}$ with $5 \% \mathrm{CO}_{2}$ ). Then, $20 \mu \mathrm{l}$ of $5 \mathrm{mg} / \mathrm{ml}$ MTT was added into each well after the removal of the culture medium. Next, the cells were incubated for an additional $4 \mathrm{~h}$. Subsequently, $150 \mu \mathrm{l}$ of DMSO was used after discarding the culture medium. Plates were placed on a plate shaker for $10 \mathrm{~min}$ and the absorbance was assessed by a microplate reader at $490 \mathrm{~nm}$.

Western blotting. Cell proteins were isolated using protein lysis buffer at $4^{\circ} \mathrm{C}$. Equal amounts of cell protein samples were separated by SDS-polyacrylamide (10\%) gel electrophoresis, and then transferred to polyvinylidene fluoride (PVDF) membranes. Then, the membranes were blocked using 5\% bovine serum albumin in Tris-buffered saline Tween-20 (TBST) for $2 \mathrm{~h}$. Subsequently, the membranes were incubated with the specific antibodies overnight, followed by incubation with a secondary antibody for $2 \mathrm{~h}$. Finally, the protein bands were exposed using ECL-detecting reagents.

Transwell assay. After Transwell chambers in 24-well plates were coated with $1 \mathrm{mg} / \mathrm{ml}$ of Matrigel matrix or not, cells were harvested and $5 \times 10^{5}$ cells $/ \mathrm{ml}$ were seeded into the Transwell chambers. The culture medium was removed after $24 \mathrm{~h}$ of incubation (at $37^{\circ} \mathrm{C}$ with $5 \% \mathrm{CO}_{2}$ ). Then, the cells were fixed with $4 \%$ paraformaldehyde for $15 \mathrm{~min}$ and stained with $1 \%$ crystal violet for another $15 \mathrm{~min}$. Subsequently, the inner-cells were wiped off with a swab and the cells were counted in random fields using a light microscope.

Cell transfection. Cells were randomly divided into 3 groups: the control, the negative control (transfected with an empty plasmid) and the shRNA-Cx32 or pcDNA-Cx32 group (transfected with Cx32-gene plasmids). According to the manufacturer's instructions, following incubation of cells in 6-well plates for $24 \mathrm{~h}$, shRNA-Cx32 or pcDNA-Cx32 genes were transfected into HepG2 or HepG2/DOX cells using Lipofectamine 2000, and then the cells were co-cultured with serum-free opti-MEM medium for $6 \mathrm{~h}$. Subsequently, the old culture was discarded and was replaced with normal culture medium.

Statistical analysis. An unpaired Student's t-test with SigmaPlot 10.0 software (Jandel Scientific, San Rafael, CA, USA) was used to assess the statistical data. Data are presented as the means \pm SEM. Differences with $\mathrm{P}<0.05$ are considered to have statistical significance. SPSS 17.0 was used to analyze the relationship between $\mathrm{Cx} 32$, E-cadherin and vimentin.

\section{Results}

Expression levels of Cx32, E-cadherin and vimentin in HCC specimens, and the corresponding paracancerous tissues. Forty patients ( 31 males and 9 females) were enrolled in the present study. The expression levels of $\mathrm{Cx} 32$, E-cadherin and vimentin in HCC tissues and adjacent non-tumorous liver samples were examined using immunohistochemistry (Fig. 1A-C). The results revealed that the expression levels of Cx32 and E-cadherin (an epithelial marker) were significantly decreased in the HCC tissues when compared to levels in the corresponding paracancerous tissues, while the expression of vimentin (a mesenchymal marker) was enhanced in the HCC specimens (Fig. 1D-F; Table I). HCC samples (55 and 40\%) showed decreased Cx32 and E-cadherin expression respectively, while $62.5 \%$ of the HCC samples showed positive expression of vimentin. 
A

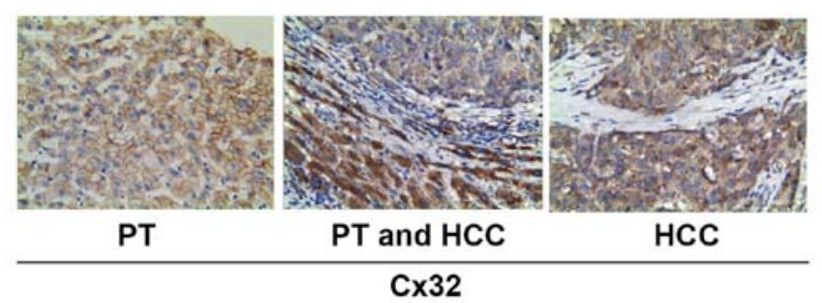

B

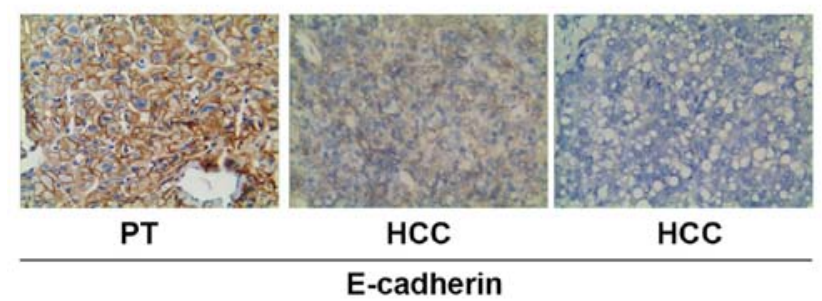

C

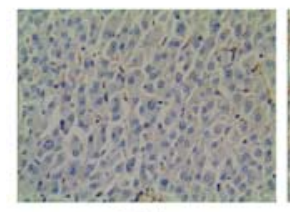

PT

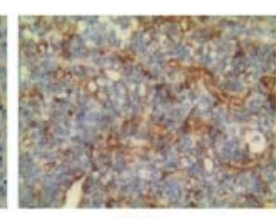

HCC
D

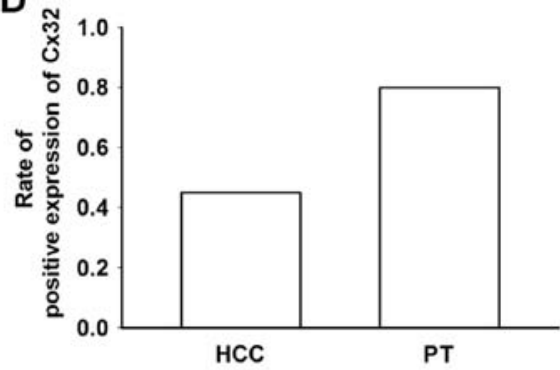

E

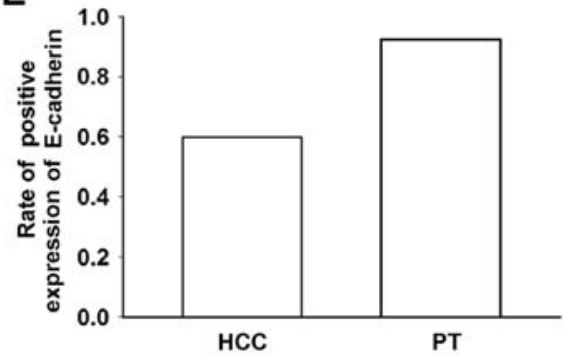

F

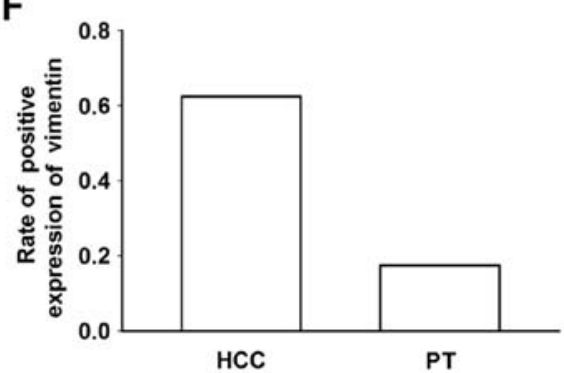

Figure 1. Expression levels of Cx32, E-cadherin and vimentin in hepatocellular carcinoma (HCC) specimens and the corresponding paracancerous tissues (PT). (A-C) The expression of Cx32, E-cadherin and vimentin were assessed using immunohistochemistry. In HCC tissues, Cx32 was mainly located in the cytoplasm, while Cx32 was expressed on the cell membrane in some paracancerous tissues. In the corresponding paracancerous tissues, E-cadherin was expressed on the cell membrane, while some HCC tissues did not express E-cadherin. In HCC, vimentin was expressed in the cytoplasm. (D-F) The rates of positive expression of Cx32, E-cadherin and vimentin in the HCC samples and the corresponding PT. Cx32, connexin 32.

Table I. Positive rates of Cx32, E-cadherin and vimentin in the HCC and paracancerous tissues.

\begin{tabular}{|c|c|c|c|c|c|c|c|c|c|c|c|c|c|c|}
\hline \multirow[b]{2}{*}{ Variant } & \multicolumn{6}{|c|}{ HCC tissues $(n=40)$} & \multicolumn{6}{|c|}{ Paracancerous tissues $(n=40)$} & \multirow[b]{2}{*}{ Fisher } & \multirow[b]{2}{*}{ P-value } \\
\hline & - & + & ++ & +++ & ++++ & $\%$ & - & + & ++ & +++ & ++++ & $\%$ & & \\
\hline Cx32 & 22 & 11 & 1 & 6 & 0 & 45.0 & 8 & 2 & 1 & 24 & 5 & 80.0 & 32.207 & $<0.001^{\mathrm{a}}$ \\
\hline E-cadherin & 16 & 11 & 8 & 5 & 0 & 60.0 & 3 & 5 & 17 & 9 & 6 & 92.5 & 46.567 & $<0.001^{\mathrm{a}}$ \\
\hline Vimentin & 15 & 3 & 14 & 5 & 3 & 62.5 & 33 & 4 & 3 & 0 & 0 & 17.5 & 11.908 & $0.046^{\mathrm{a}}$ \\
\hline
\end{tabular}

${ }^{\mathrm{a}} \mathrm{P}<0.05$ vs. paracancerous tissues. Cx32, connexin $32 ; \mathrm{HCC}$, hepatocellular carcinoma.

Spearman correlation analysis was used to observe the relationship between $\mathrm{Cx} 32$, E-cadherin and vimentin in the HCC samples. Table II shows that there is a strong correlation between Cx32, E-cadherin and vimentin in HCC tissues. The expression level of E-cadherin was positively correlated with the expression of $\mathrm{Cx} 32$, while the expression of vimentin was negatively correlated with the expression of $\mathrm{Cx} 32$.

HepG2/DOX cell line is established. The HepG2/DOX cell line was induced via serially increasing DOX concentrations.
An MTT assay was used to investigate the cytotoxicity of DOX in HepG2 cells (sensitive to DOX) and HepG2/DOX (resistant to DOX) cells. The results in Fig. 2 showed that the $\mathrm{IC}_{50}$ value of DOX in the HepG2 cells was $0.182 \mu \mathrm{g} / \mathrm{ml}$, while the $\mathrm{IC}_{50}$ value of DOX in the HepG2/DOX cells was $2.058 \mu \mathrm{g} / \mathrm{ml}$. Thus, the HepG2/DOX cells demonstrated an 11.31-fold higher resistance to DOX than the HepG2 cells.

HepG2/DOX cells undergo EMT. The expression of major markers of EMT in the HepG2 cells and HepG2/DOX cells 


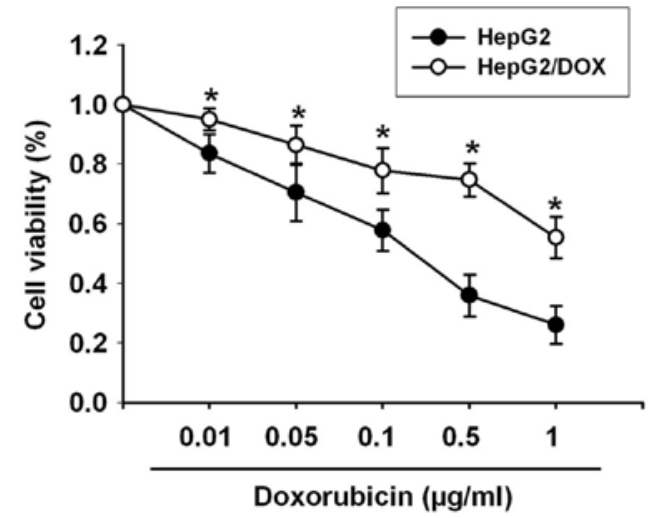

Figure 2. DOX-resistant cell line was established from the parental cell line HepG2. HepG2 and HepG2/DOX cells were exposed to different concetrations of DOX $(0.01,0.05,0.1$ and $0.5,1)$ for $24 \mathrm{~h}$. Then, the cell viability was detected by MTT assay; ${ }^{\mathrm{P}}<0.05$ vs. the HepG2 group. DOX, doxorubicin.

were observed by western blotting. Results revealed that epithelial marker E-cadherin was significantly decreased in the HepG2/DOX cells compared with that in the HepG2 cells. In contrast, the expression level of mesenchymal marker vimentin
Table II. Correlation analysis of $\mathrm{Cx} 32$, E-cadherin and vimentin in the HCC samples.

\begin{tabular}{lcr}
\hline & \multicolumn{2}{c}{$\mathrm{Cx32}$} \\
\cline { 2 - 3 } Variables & Spearman indices & P-value \\
\hline E-cadherin & 0.812 & $<0.001^{\mathrm{a}}$ \\
Vimentin & -0.502 & $0.001^{\mathrm{a}}$
\end{tabular}

${ }^{\mathrm{a}} \mathrm{P}<0.05$ vs. Cx32. Cx32, Connexin 32; HCC, hepatocellularcarcinoma.

was clearly increased in the HepG2/DOX cells (Fig. 3A-C). In order to further observe the changes of EMT in HepG2/DOX cells, the invasive and migratory abilities of the HepG2/DOX and HepG2 cells were detected by Transwell assays. Fig. 3D-F revealed that compared to the HepG2 cells, the HepG2/DOX cells demonstrated higher invasion and migration potential. This result suggests that acquired resistance could enhance the invasion and migration abilities of HepG2 cells. All the aforementioned results demonstrated that the HepG2/DOX cells acquired an EMT phenotype.
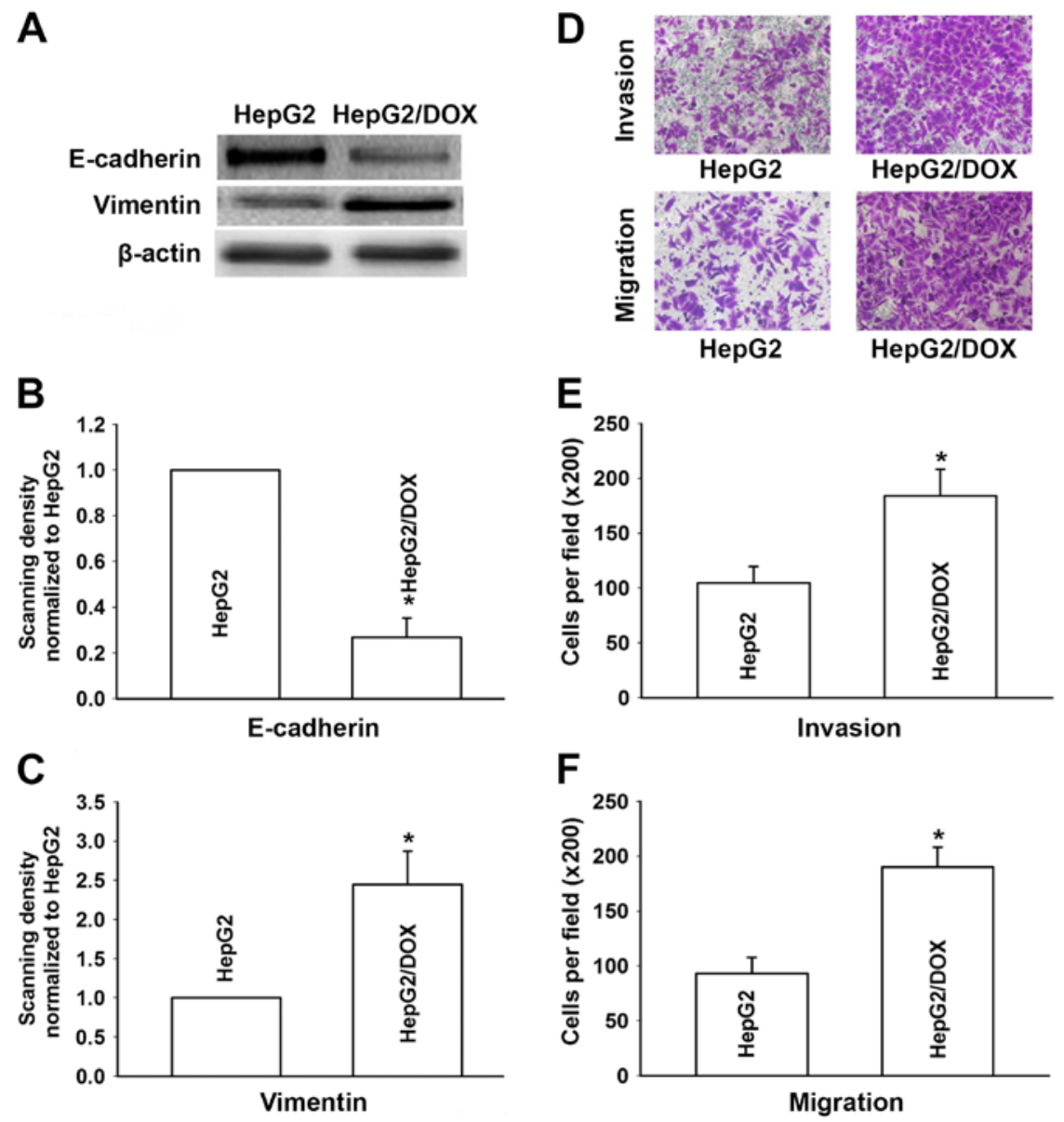

Figure 3. HepG2/DOX cells exhibit EMT characteristics. (A) Expression levels of E-cadherin and vimentin in the HepG2 and HepG2/DOX cells were observed by western blotting. (B and C) Bar graphs are derived from the densitometric scanning results. The results represent the means \pm SEM of 3 independent experiments; "P<0.05 vs. the HepG2 group. (D) The invasion and migration abilities of HepG2 and HepG2/DOX cells were examined by Transwell assay. (E and F) Bar graphs are derived from the results of the Transwell assays. Results represent the means \pm SEM of 3 independent experiments; ${ }^{*} \mathrm{P}<0.05$ vs. the HepG2 group. DOX, doxorubicin; EMT, epithelial-mesenhymal transition. 


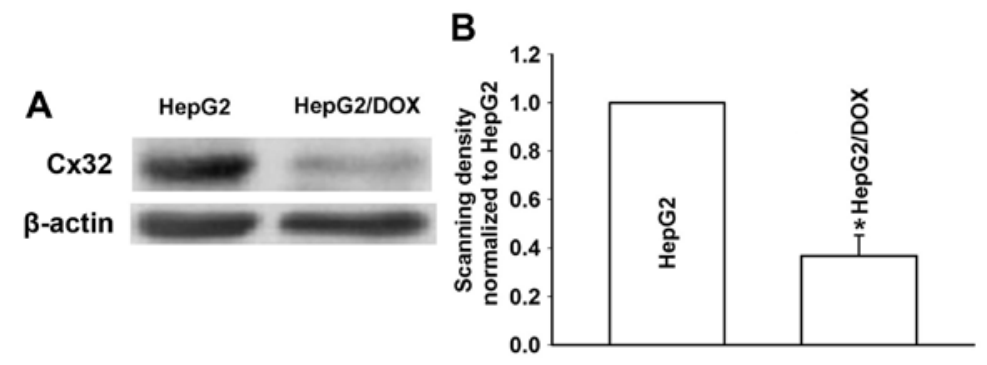

Figure 4. (A-B) Cx32 was significantly decreased in the HepG2/DOX cells when compared with the HepG2 cells. The expression level of Cx32 was detected by western blotting; " $\mathrm{P}<0.05$ vs. the HepG2 group. DOX, doxorubicin.
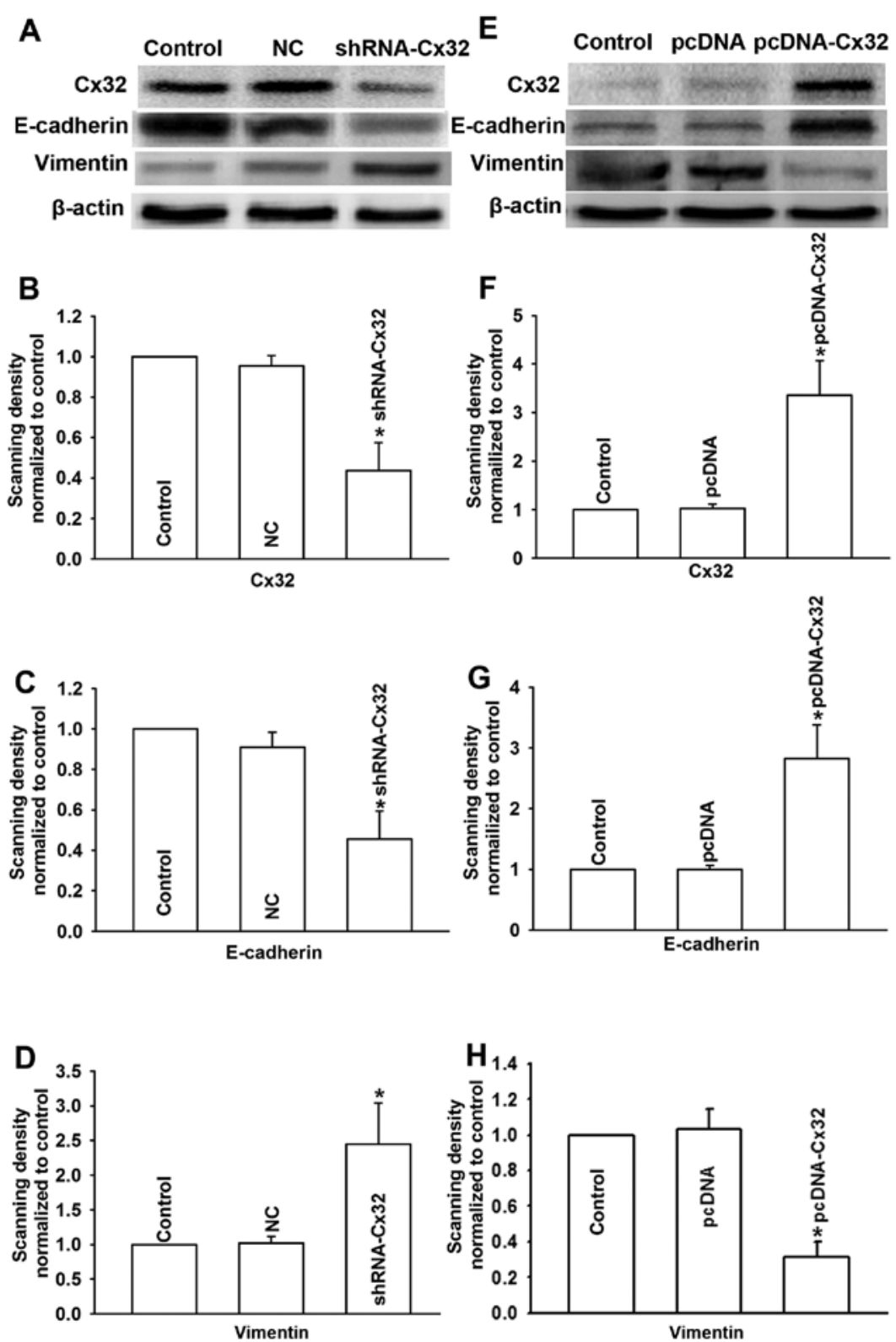

Figure 5. Cx32 regulates EMT in HCC. (A) The expression levels of E-cadherin and vimentin were observed after decreasing Cx32 by shRNA-Cx32 in HepG2 cells. (B-D) Results represent the means \pm SEM from 3 independent experiments; " $\mathrm{P}<0.05$ vs. the negative control (NC) group. (E) The expression levels of E-cadherin and vimentin were examined after the transfection of HepG2/DOX cells with pcDNA-Cx32. (F-H) Results represent the means \pm SEM from 3 independent experiments; " $\mathrm{P}<0.05$ vs. the pcDNA group. EMT, epithelial-mesenchymal transition; HCC, hepatocellular carcinoma; DOX, doxorubicin.

Acquired resistance of DOX decreases the expression of $C \times 32$. We investigated the expression of $\mathrm{Cx} 32$ by western blotting in HepG2 and HepG2/DOX cells. As shown in Fig. 4, the HepG2 cells showed a significantly increased expression level of Cx32 compared with that in the HepG2/DOX cells. This result demonstrated that $\mathrm{Cx} 32$ may play an important 
A

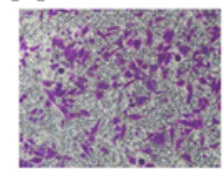

control

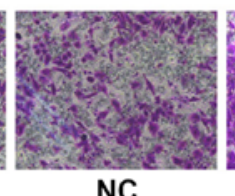

NC

B

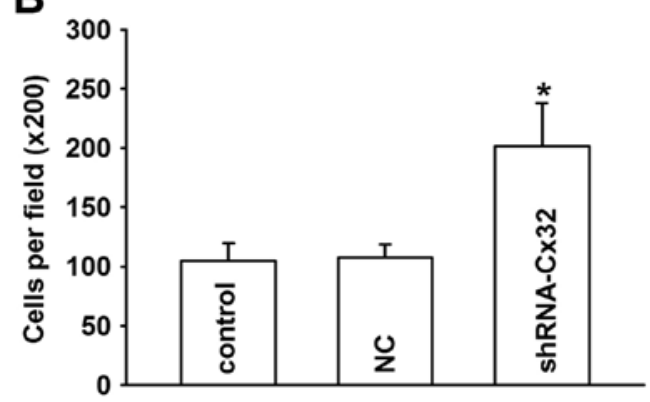

E

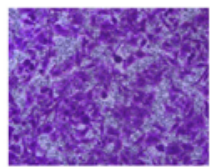

control

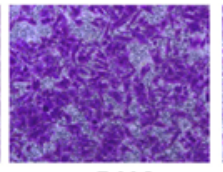

pcDNA

F

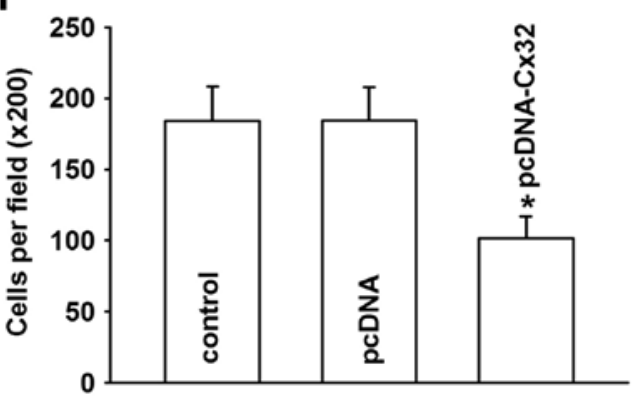

C

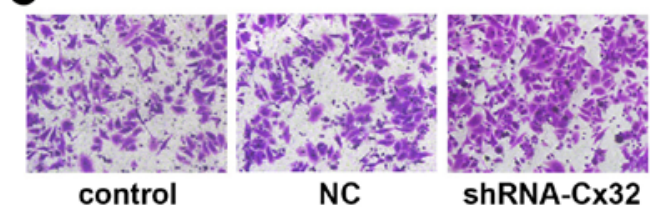

D

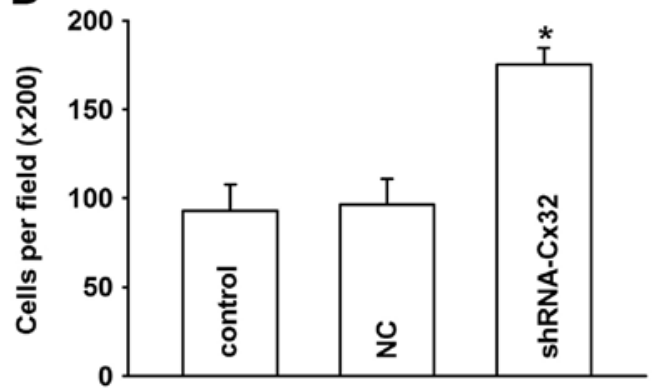

G

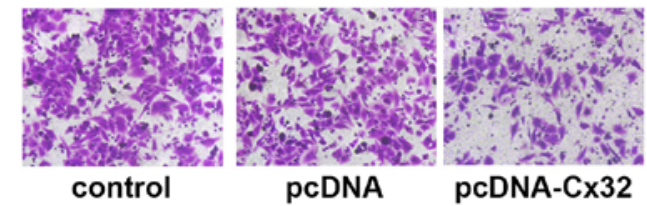

H

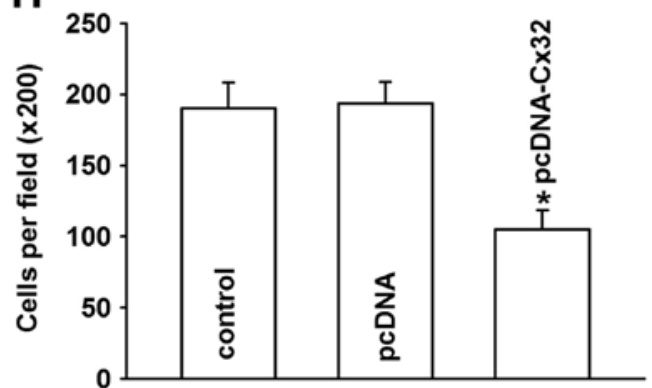

Figure 6. Cx32 affects the ability of invasion and migration in HepG2 and HepG2/DOX cells. (A and C) The invasive and migratory abilities were clearly enhanced after decreasing Cx32 by shRNA-Cx32 in the HepG2 cells. (B and D) Bar graphs are derived from the results of Transwell assays. The results represent the means \pm SEM from 3 independent experiments; ${ }^{*} \mathrm{P}<0.05$ vs. The negative control (NC) group. $(\mathrm{E}$ and $\mathrm{G})$ The invasive and migratory abilities were observed after the transfection of HepG2/DOX cells with pcDNA-Cx32. (F and H) Bar graphs represent the means \pm SEM from 3 independent experiments; ${ }^{*} \mathrm{P}<0.05$ vs. the pcDNA group. Cx32, connexin 32; DOX, doxorubicin.

role in the mechanism of acquired resistance of HepG2 cells to DOX.

\section{Cx32 regulates EMT in $H C C$}

Inhibition of Cx32 induces EMT in HepG2 cells. The aforementioned results showed that the HepG2/DOX cells initiated EMT with decreased expression of Cx32. Then, we observed the effect of Cx32 on DOX-induced EMT in two ways: inhibition of $\mathrm{Cx} 32$ expression with shRNA-Cx32 in HepG2 cells, and overexpression of $\mathrm{Cx} 32$ with pcDNACx32 in HepG2/DOX cells.

First, we decreased the expression of $\mathrm{Cx} 32$ in $\mathrm{HepG} 2$ cells by shRNA-Cx32. As shown in Fig. 5A and B, after transfecting the HepG2 cells with shRNA-Cx32, the expression of Cx32 was significantly decreased in the shRNA-Cx32 group when compared with the control or negative control group. Then, to explore the effect of $\mathrm{Cx} 32$ on EMT in HepG2 cells, we also detected the expression of EMT markers (E-cadherin and vimentin) by western blotting as well as the invasion and migration abilities of the cells by Transwell assays. The results in Fig. 5A and $\mathrm{C}$ demonstrated that the expression of E-cadherin in the shRNA-Cx32 group was clearly decreased when compared with the control or negative control group. Inversely, vimentin was significantly enhanced (Fig. 5A and D). The results of Transwell assays also revealed that the HepG2 cells acquired enhanced invasive and migratory abilities after the inhibition of the expression of $\mathrm{Cx} 32$ (Fig. 6A and D). These results suggest that downregulation of $\mathrm{Cx} 32$ by shRNA-Cx32 induced EMT in the HepG2 cells, which indicated that $\mathrm{Cx} 32$ is partly responsible for the initiation of EMT in HepG2 cells.

Overexpression of Cx32 converts EMT to mesenchymal-epithelial transition (MET) in HepG2/DOX cells. To further observe the role of $\mathrm{C} \times 32$ in the mechanism of DOX-induced EMT, we used a $\mathrm{Cx} 32$ gene fragment to overexpress $\mathrm{C} \times 32$ in HepG2/DOX cells which were resistant to DOX. After HepG2/DOX cells were transfected with pcDNA-Cx32 for $24 \mathrm{~h}$, the expression level of Cx32 was detected by western 
blotting. The result revealed that the pcDNA-Cx32 group showed significantly increased expression of Cx32 when compared to that of the control or the negative control group (Fig. 5E and F). To explore the effect of the upregulation of Cx32 on EMT in HepG2/DOX cells, EMT markers E-cadherin and vimentin as well as the invasion and migration abilities of the cells were also examined. As shown in Fig. 5E and $\mathrm{G}, \mathrm{E}$-cadherin was significantly enhanced in the pcDNACx32 group, while vimentin was obviously decreased (Fig. 5E and $\mathrm{H}$ ). The invasion and migration abilities also decreased after transfecting HepG2/DOX cells with Cx32 (Fig. 6E-H). These findings indicate that overexpression of $\mathrm{Cx} 32$ reverses EMT to MET in HepG2/DOX cells

\section{Discussion}

In recent years, studies have focused on the role of EMT in the acquired resistance of chemotherapeutics drugs (16,17-20), and acquisition of EMT has recently been proposed as an important mechanism for drug resistance in cancer cells. For example, cisplatin-resistance in lung cancer cells, cisplatin-resistance in cervical cancer cells, crizotinib-resistant anaplastic lymphoma kinase (ALK)-positive lung cancer cells, gemcitabine-resistant hepatoma cells, tamoxifen-resistant MCF7 breast cancer cells all possess EMT characteristics. Recently, targeting EMT has been considered as a new strategy to combat cancer drug resistance (21). In the present study, we also found that during the process of acquisition of DOX resistance, HepG2/DOX cells exhibited an EMT phenotype.

Cxs have been reported to be the components of gap-junctional communication channels and important tumor suppressors $(9,10,22)$. Recently, 21 connexins and related genes have been found in the human body (23), among them, Cx43, Cx26 and Cx32 have the most extensive distribution. Normal hepatocytes express $\mathrm{Cx} 32$ and to a lesser extent $\mathrm{Cx} 26$, which represent $\sim 90$ and 5\%, respectively, of the total $\mathrm{Cx}$ amount in rat and human livers. Cx32 is uniformly distributed throughout the liver, while Cx26 is mainly expressed in the periportal acinar area (24). During the process of hepatocarcinogenesis, both Cx32 and Cx26 are decreased (24). In the present study, Cx26 was not detected in the HepG2 and HepG2/DOX cells (data not shown), while the aforementioned 2 cell lines expressed different levels of $\mathrm{Cx} 32$. Thus, the research focused on the effect of Cx32, but not Cx26, on DOX-induced EMT. Reports showed that Cx32 demonstrated a suppressive effect on liver inflammation, fibrosis, hepatocarcinogenesis and hepatoma cell metastasis (25-28). Nakashima et al also found that the expression of $\mathrm{Cx} 32$ in hepatocellular carcinoma (HCC) patients was significantly decreased compared with the non-HCC patients (27). Cx32 overexpression decreased the malignant phenotype of liver tumors $(29,30)$. The present study reveals a new role of $\mathrm{Cx} 32$ in the regulation of cancer metastasis and drug resistance in HCC.

In our previous study (16), we found that cisplatin (CDDP)resistant cell line A549/CDDP acquired an EMT phenotype. Overexpression of $\mathrm{Cx} 43$ converted EMT to MET and enhanced the sensitivity of CDDP to A549/CDDP cells. The results revealed that $\mathrm{Cx} 43$ was correlated with EMT in A549/CDDP cells. The present study further investigated the relationship between Cx and EMT in chemotherapy drug-resistant cells.
We used HepG2 cells to establish the DOX-resisistant cell line. The present study demonstrated that when the HepG2 cells became resistant to DOX, the HepG2 cells acquired an EMT phenotype, meanwhile, Cx32 was significantly decreased in the HepG2/DOX cells. We also found that downregulation of Cx32 by shRNA-Cx32 induced EMT in the HepG2 cells, while overexpression of Cx 32 reversed EMT in the HepG2/DOX cells. Thus, the results in HCC were consistent with the results in human lung adenocarcinoma, which suggest that Cx-induced EMT may be responsible for drug resistance in cancer cells and $\mathrm{Cx}$ is an important target for overcoming drug resistance when cells initiate EMT.

Cx hemichannels can supply a pathway for cellular signaling between cells and their extracellular environment (31-33). The messengers that diffuse through $\mathrm{Cx}$ hemichannels usually include adenosine triphosphate (ATP), glutamate and glutathione. Studies have shown that both ATP and glutathione participate in the regulation of EMT in different cells (34-39). Thus, an inference is that ATP and/or glutathione may be responsible for Cx32-mediated EMT in HCC. Studies may be performed in the future to observe the role of ATP and glutathione in Cx32-mediated EMT in HCC.

In summary, the present study is the first to establish the role of Cx32 in the occurrence of EMT in liver carcinoma cells. We demonstrated that the upregulation of the expression of Cx32 in HepG2/DOX cells inhibited EMT and enhanced DOX-induced cytotoxicity. Therefore, Cx32 may be a new target to potentiate DOX cytotoxicity in hepatic carcinoma in the future.

\section{Acknowledgements}

The present study was supported by the National Natural Science Foundation of Anhui (grant no. 1508085QH151) and the Natural Science Foundation of the Provincial Education Department of Anhui (no. KJ2015A147), the visiting project of the Provincial Education Department of Anhui (gxfxZD2016142), the National Natural Science Foundation of China (no. 81001457), and the Foundation of Bengbu Medical College (nos. Byycxz1422 and Byky1407ZD).

\section{References}

1. Siegel R, Naishadham D and Jemal A: Cancer statistics, 2012. CA Cancer J Clin 62: 10-29, 2012.

2. Zhu AX: Systemic therapy of advanced hepatocellular carcinoma: How hopeful should we be? Oncologist 11: 790-800, 2006.

3. Asghar U and Meyer T: Are there opportunities for chemotherapy in the treatment of hepatocellular cancer? J Hepatol 56: 686-695, 2012.

4. Xu F, Wang F, Yang T, Sheng Y, Zhong T and Chen Y: Differential drug resistance acquisition to doxorubicin and paclitaxel in breast cancer cells. Cancer Cell Int 14: 538, 2014.

5. Harada K, Ferdous T and Ueyama Y: Establishment of 5-fluorouracil-resistant oral squamous cell carcinoma cell lines with epithelial to mesenchymal transition changes. Int J Oncol 44: 1302-1308, 2014.

6. Haider M, Zhang X, Coleman I, Ericson N, True LD, Lam HM, Brown LG, Ketchanji M, Nghiem B, Lakely B, et al: Epithelial mesenchymal-like transition occurs in a subset of cells in castration resistant prostate cancer bone metastases. Clin Exp Metastasis 33: 239-248, 2016

7. Xu H, Pirisi L and Creek KE: Six1 overexpression at early stages of HPV16-mediated transformation of human keratinocytes promotes differentiation resistance and EMT. Virology 474: 144-153, 2015. 
8. Dinicola S, Pasqualato A, Proietti S, Masiello MG, Palombo A Coluccia P, Canipari R, Catizone A, Ricci G, Harrath AH, et al: Paradoxical E-cadherin increase in 5FU-resistant colon cancer is unaffected during mesenchymal-epithelial reversion induced by $\gamma$-secretase inhibition. Life Sci 145: 174-183, 2016.

9. Zhou JZ and Jiang JX: Gap junction and hemichannel-independent actions of connexins on cell and tissue functions - an update. FEBS Lett 588: 1186-1192, 2014.

10. Aasen T: Connexins: Junctional and non-junctional modulators of proliferation. Cell Tissue Res 360: 685-699, 2015.

11. Jiang JX and Gu S: Gap junction- and hemichannel-independent actions of connexins. Biochim Biophys Acta 1711: 208-214, 2005.

12. Diezmos EF, Bertrand PP and Liu L: Purinergic signaling in gut inflammation: The role of connexins and pannexins. Front Neurosci 10: 311, 2016.

13. Cronier L, Crespin S, Strale PO, Defamie N and Mesnil M: Gap junctions and cancer: New functions for an old story. Antioxid Redox Signal 11: 323-338, 2009.

14. Kandouz M and Batist G: Gap junctions and connexins as therapeutic targets in cancer. Expert Opin Ther Targets 14: 681-692, 2010.

15. Trosko JE and Ruch RJ: Gap junctions as targets for cancer chemoprevention and chemotherapy. Curr Drug Targets 0.3 . 465-482, 2002.

16. Yu M, Zhang C, Li L, Dong S, Zhang N and Tong X: Cx43 reverses the resistance of A549 lung adenocarcinoma cells to cisplatin by inhibiting EMT. Oncol Rep 31: 2751-2758, 2014.

17. Song $\mathrm{J}$ and $\mathrm{Li} \mathrm{Y}$ : miR-25-3p reverses epithelial-mesenchymal transition via targeting Sema4C in cisplatin-resistance cervical cancer cells. Cancer Sci: Oct 15, 2016 (Epub ahead of print) doi: $10.1111 /$ cas. 13104.

18. Gao HX, Yan L, Li C, Zhao LM and Liu W: miR-200c regulates crizotinib-resistant ALK-positive lung cancer cells by reversing epithelial-mesenchymal transition via targeting ZEB1. Mol Med Rep 14: 4135-4143, 2016.

19. Wu Q, Wang R, Yang Q, Hou X, Chen S, Hou Y, Chen C, Yang Y, Miele L, Sarkar FH, et al: Chemoresistance to gemcitabine in hepatoma cells induces epithelial-mesenchymal transition and involves activation of PDGF-D pathway. Oncotarget 4: 1999-2009, 2013.

20. Hiscox S, Jiang WG, Obermeier K, Taylor K, Morgan L, Burmi R, Barrow D and Nicholson RI: Tamoxifen resistance in MCF7 cells promotes EMT-like behaviour and involves modulation of $\beta$-catenin phosphorylation. Int J Cancer 118: 290-301, 2006.

21. Du B and Shim JS: Targeting epithelial-mesenchymal transition (EMT) to overcome drug resistance in cancer. Molecules 21: 21, 2016.

22. Kay CW, Ursu D, Sher E and King AE: The role of Cx36 and Cx43 in 4-aminopyridine-induced rhythmic activity in the spinal nociceptive dorsal horn: An electrophysiological study in vitro. Physiol Rep 4: 4, 2016.

23. Unger VM, Kumar NM, Gilula NB and Yeager M: Threedimensional structure of a recombinant gap junction membrane channel. Science 283: 1176-1180, 1999.

24. Maes M, Decrock E, Cogliati B, Oliveira AG, Marques PE, Dagli ML, Menezes GB, Mennecier G, Leybaert L, Vanhaecke T et al: Connexin and pannexin (hemi)channels in the liver. Front Physiol 4: 405, 2014.

25. De Maio A, Gingalewski C, Theodorakis NG and Clemens MG: Interruption of hepatic gap junctional communication in the rat during inflammation induced by bacterial lipopolysaccharide. Shock 14: 53-59, 2000.
26. Sagawa H, Naiki-Ito A, Kato H, Naiki T, Yamashita Y, Suzuki S, Sato S, Shiomi K, Kato A, Kuno T, et al: Connexin 32 and luteolin play protective roles in non-alcoholic steatohepatitis development and its related hepatocarcinogenesis in rats. Carcinogenesis 36 : 1539-1549, 2015.

27. Nakashima Y, Ono T, Yamanoi A, El-Assal ON, Kohno H and Nagasue N: Expression of gap junction protein connexin32 in chronic hepatitis, liver cirrhosis, and hepatocellular carcinoma. J Gastroenterol 39: 763-768, 2004.

28. Zhao B, Zhao W, Wang Y, Xu Y, Xu J, Tang K, Zhang S, Yin Z, Wu Q and Wang X: Connexin32 regulates hepatoma cell metastasis and proliferation via the p53 and Akt pathways. Oncotarget 6: 10116-10133, 2015.

29. Yang J, Ichikawa A and Tsuchiya T: A novel function of connexin 32: Marked enhancement of liver function in a hepatoma cell line. Biochem Biophys Res Commun 307: 80-85, 2003.

30. Muramatsu A, Iwai M, Morikawa T, Tanaka S, Mori T, Harada Y and Okanoue T: Influence of transfection with connexin 26 gene on malignant potential of human hepatoma cells. Carcinogenesis 23: 351-358, 2002.

31. Decrock E, Vinken M, De Vuyst E, Krysko DV, D Herde K, Vanhaecke T, Vandenabeele P, Rogiers V and Leybaert L: Connexin-related signaling in cell death: To live or let die? Cell Death Differ 16: 524-536, 2009.

32. Chandrasekhar A and Bera AK: Hemichannels: Permeants and their effect on development, physiology and death. Cell Biochem Funct 30: 89-100, 2012

33. Kar R, Batra N, Riquelme MA and Jiang JX: Biological role of connexin intercellular channels and hemichannels. Arch Biochem Biophys 524: 2-15, 2012.

34. Unahabhokha T, Chanvorachote P, Sritularak B Kitsongsermthon J and Pongrakhananon V: Gigantol Inhibits Epithelial to Mesenchymal Process in Human Lung Cancer Cells. Evid Based Complement Alternat Med 2016: 4561674, 2016.

35. Park SJ, Choi YS, Lee S, Lee YJ, Hong S, Han S and Kim BC: BIX02189 inhibits TGF- $\beta 1$-induced lung cancer cell metastasis by directly targeting TGF- $\beta$ type I receptor. Cancer Lett 381: 314-322, 2016.

36. Zhou Q, Abraham AD, Li L, Babalmorad A, Bagby S, Arcaroli JJ, Hansen RJ, Valeriote FA, Gustafson DL, Schaack J, et al: Topoisomerase II $\alpha$ mediates TCF-dependent epithelial-mesenchymal transition in colon cancer. Oncogene 35: 4990-4999, 2016.

37. Wang HJ, Zhu J and Zheng GY: Role of glutathione and other antioxidants in the inhibition of apoptosis and mesenchymal transition in rabbit lens epithelial cells. Genet Mol Res 13: 7149-7156, 2014.

38. Zhang J, Qiu M, Ma Y, Bu Y, Yang L and Tang X: Advanced oxidation protein products induce epithelial-to-mesenchymal transition in cultured human proximal tubular epithelial cells via oxidative stress. Nan Fang Yi Ke Da Xue Xue Bao 34: 659-663, 2014 (In Chinese)

39. Ryoo IG, Shin DH, Kang KS and Kwak MK: Involvement of Nrf2-GSH signaling in TGF 31 -stimulated epithelial-to-mesenchymal transition changes in rat renal tubular cells. Arch Pharm Res 38: 272-281, 2015. 